\title{
Food Allergy: Clinical Management for General Pediatricians
}

\section{Maysoun Al-Rushood}

Pediatric allergy and clinical immunology, Department of pediatrics, Kuwait University, 24923, Safat 13110, Kuwait

\section{Introduction}

Food adverse reactions consist of any abnormal reaction after ingestion of food. These can be ranged from life threatening anaphylaxis to milder reactions such as diarrhea or abdominal cramps. They could be classified as immune-mediated and nonimmune-mediated. It is important to differentiate between the two as the management, prognosis and risks vary.

Lactose intolerance, which is a metabolic disorder characterized by the inability to digest lactose due to the lack of the enzyme lactase, is occasionally misinterpreted as food allergy. Toxic contamination may mimic food allergy, such as scombroid fish poisoning.

Food allergy is defined as an adverse reaction arising from a specific immune response that occurs reproducibly on exposure to given food [1]. These could be IgE mediated or non-IgE mediated. This review focuses on type I hypersensitivity reaction, which is an IgE-mediated.

The aim of this article is to elicit the importance of accurately diagnose food allergy. It has been shown that such diagnosis negatively affects the quality of life of the patients as well as their families. There is increased anxiety to avoid accidental ingestion $[2,3]$. Moreover, overdiagnosis could lead to unnecessary dietary restrictions compromising the health and growth of patients, especially young infants. On the other hand, underdiagnosis could lead to life threatening anaphylaxis upon exposure to the culprit food.

\section{Epidemiology}

Several studies have illustrated the overall increased prevalence of food allergy $[2,4,5]$. In the United States, food allergy is thought to occur in approximately $6-8 \%$ of children [2,5]. However, accurate determination of the incidence and prevalence is influenced by several methodological problems and study limitations [2,5]. Studies are not uniform in the definition of food allergy, study population, geographic differences, and methods of diagnosis. Few studies conducted doubleblind, placebo-controlled food challenges (DBPCFC) which are the gold standard for the diagnosis. Many studies are based on self-report, specific IgE levels and/or skin prick test results, which might reflect sensitization depending on the correlation with the medical history.

Generally, the most common food allergens in pediatrics are milk, egg, soy and wheat. Other important allergens to consider are tree nut, peanut, fish and shellfish.

The majority of children outgrow their milk, egg, soy and wheat allergy during their childhood [2,5] About 80\% outgrow their milk allergy by their fifth birthday [6]. Yet, recent studies have shown that the rate of resolution has slowed.

On the other hand peanut and tree nut allergies tend to be persistent; with only $20 \%$ of children outgrow their allergies.

\section{Clinical diagnosis}

The symptoms of type I hypersensitivity reaction are mostly related to the acute mast cell degranulation and the release of its mediators, of which, histamine plays the major role. They typically occur immediately to few minutes or hours after ingestion. They resolve spontaneously with or without treatment upon removal of the causative agent.

\section{Publication History:}

Received: March 24, 2015

Accepted: June 06, 2015

Published: June 08, 2015

\section{Keywords:}

Food allergy, Clincical management, Epidemiology, Clicnical diagnosis

The majority of the reactions involve skin manifestations, such as pruritus, urticaria and angioedema. Other systems might be involved in more severe reactions. It is essential to be able to recognize anaphylaxis. Table 1 summarizes the clinical criteria for the diagnosis of anaphylaxis according to the World Allergy Organization (WAO) guidelines. Upon exposure to a known allergen, a drop of the blood pressure could be the only clinical sign of anaphylaxis, or in similar example, generalized urticaria might be the only initial manifestation of anaphylaxis [7].

It is critical to obtain detailed history to determine the foods that are more likely to cause the reaction. Important information include the food ingested, its quantity, preparation of the food, whether it is processed, food additives, the length of time between ingestion and development of symptoms, the last reaction if any, any activity proximate to the ingestion, any medication taken, any comorbidities such as asthma.

Diet diary is helpful when the culprit food cannot be identified. It is also important to know that eating in restaurants increases the risk of being unintentionally exposed to the allergen either as a hidden or unidentified food. Cross-contact refers to inadvertent transfer fro a food that contains the allergen to a food that does not. It occurs during food preparation or packaging. It is more common at a buffet, bakery, Asian (commonly uses tree nut and peanut products) or seafood restaurants and with deep-fried food (the oil may be reused for different food) [2].

Instructions to completely avoid the possible culprit foods until seen by an allergist must be given to the patient. This indicates reading food labels and avoid cross-contact. It should be clear to the patients and their families that they should not try the suspected food at home as the severity of subsequent reactions cannot be predicted. Emergency action plan should be discussed with the patient. The details of the management will be discussed later in this review.

After evaluation, patients should be referred to an allergist for proper diagnosis and management. Interpretation of allergic test results should be carefully done based on clinical data. Sensitization does not lead to clinical reactivity in all patients. False negative results should be considered, as squeals may be fatal. (See later).

${ }^{\overline{ }}$ Corresponding Author: Dr. Maysoun Al-Rushood, Pediatric allergy and clinical immunology, Department of pediatrics, Kuwait University, 24923, Safat 13110, Kuwait; E-mail: maysounrushood@hsc.edu.kw

Citation: Al-Rushood M (2015) Food Allergy: Clinical Management for General Pediatricians. Int J Pediatr Neonat Care 1: 107. doi: http://dx.doi. org/10.15344/2455-2364/2015/107

Copyright: (C) 2015 Al-Rushood. This is an open-access article distributed under the terms of the Creative Commons Attribution License, which permits unrestricted use, distribution, and reproduction in any medium, provided the original author and source are credited. 
Anaphylaxis is highly likely when any one of the following three criteria is fulfilled

1. Acute onset of an illness (minutes to several hours) with involvement of the skin, mucosal tissue, or both (eg, generalized urticaria, itching or flushing, swollen lips-tongue-uvula) AND AT LEAST ONE OF THE FOLLOWING: A) Respiratory compromise (eg, dyspnea, wheezebronchospasm, stridor, reduced PEF, hypoxemia) B) Reduced blood pressure or associated symptoms of end-organ dysfunction (eg. Hypotonia, collapse_, syncope, incontinence) OR

2. Two or more of the following that occur rapidly after exposure to a likely allergen (a)for that patient (minutes to several hours)

A) Involvement of the skin-mucosal tissue (eg, generalized urticaria, itch-flush, swollen lips-tongue-uvula)

B) Respiratory compromise (eg, dyspnea, wheezebronchospasm, stridor, reduced PEF, hypoxemia) C) Reduced blood pressure or associated symptoms (eg, hypotonia_collapse_, syncope, incontinence)

D) Persistent gastrointestinal symptoms (eg, crampy abdominal pain, vomiting) OR

3. Reduced blood pressure after exposure to known allergen (b)for that patient (minutes to several hours)

A) Infants and children: low systolic blood pressure (agespecific) or greater than $30 \%$ decrease in systolic blood pressure(c)

B) Adults: systolic blood pressure of less than $90 \mathrm{~mm} \mathrm{Hg}$ or greater than $30 \%$ decrease from that person's baseline PEF: peak expiratory flow.

(a)Or other trigger, for example, immunologic but IgE-independent, or nonimmunologic (direct) mast cell activation.

(b)For example, after an insect sting, reduced blood pressure might be the only manifestation of anaphylaxis; or, in a similar example, during allergen immunotherapy, after injectionof a known allergen for that patient, generalized urticaria (only one body organ system affected) might be the only initial manifestation of anaphylaxis.

(c)Low systolic blood pressure for children is defined as less than $70 \mathrm{~mm} \mathrm{Hg}$ from 1 month to 1 year, less than (70 $\mathrm{mm} \mathrm{Hg}+(2 \mathrm{x}$ age $))$ from 1 to 10 years, and less than 90 $\mathrm{mm} \mathrm{Hg}$ from 11to 17 years. Normal heart rate ranges from $80-140$ beats/min at age $1-2$ years; from $80-120$ beats/min at age 3 years; and from $70-115$ beats/min after age 3 years. Infants are more likely to haverespiratory compromise than hypotension or shock, and in this age group, shock is more likely to be manifest initially by tachycardia than by hypotension.

Table 1: Clinical Criteria for Diagnosing Anaphylaxis.

\section{Skin prick test (SPT)}

This is the primary diagnostic method done by most allergists. It involves applying food extracts or fresh food to the skin (typically the volar aspect of the forearm) and the skin is pricked with an instrument [8].
SPT can be performed in the office. It is safe, highly sensitive, the results are readily available and a wide range of allergens can be tested in one setting.

A positive result might reflect sensitization, not necessarily clinical reactivity or disease severity, especially if it does not support the history or in the absence of prior exposure.

A negative SPT is of particular importance, as it has high negative predictive value. It is useful to rule out allergy. Again, this does not guarantee clinical tolerance. When the suspicion is high, further evaluation should be performed. Therefore, it is crucial to interpret the results based on overall clinical evaluation.

\section{Serum specific IgE tests}

Serum specific IgE is another important tool for diagnosis of food allergy. It is particularly important to perform when the SPT cannot be conducted. Patients, who cannot discontinue antihistamines, or with extensive skin disease or who have significant dermatographism are candidates for this test. It is also useful when the SPT results are equivocal. It reflects sensitization and not clinical outcome. However, the predictive values for limited number of foods have been established $[2,5]$.

\section{Oral food challenge (OFC)}

A food challenge involves feeding the patient incremental amounts of the culprit food and observing for clinical reaction [8]. These could be conducted as DBPCFC, single blind with or without placebo or open.

The gold standard for the diagnosis of food allergy is DBPCFC. However, these are labor and time consuming, expensive and there is a risk of anaphylaxis, which could lead to death. It is for all these reasons; they are not performed routinely for the diagnosis. On the contrary, they are performed to monitor for resolution when, based on clinical and lab data, the probability of allergic reaction is very low.

OFC is not necessary if the patient's history is convincing with positive specific IgE testing (SPT or serum). The food is known from clinical evaluation. In addition, the history should take priority in the decision making over the lab findings.

However, on occasions, OFCs are the only means for diagnosis, when the history is equivocal and the results of IgE are negative. The decision should be made with caution, taking into account the risks and the benefits. It should be conducted in the appropriate clinical setting under the supervision of trained medical staff to deal with anaphylaxis.

\section{Component resolved diagnostic testing (CRD)}

CRD is a novel method for diagnosis. It was proposed about 15 years ago and recently enters medical practice [5,9]. It identifies the patient's specific IgE reactivity recombinant allergenic protein rather than the whole protein [2]. The details and the application of this test are beyond the scope of this review.

\section{Others}

Serum IgG level has been increasingly used by different practitioners to diagnose food allergy. It should be noted that these tests are not diagnostic. Allergists do not use them because increased levels of food specific IgG may indicate tolerance rather than allergy [8]. This test is not recommended as it is nonstandardized and unproved [5]. 
Citation: Al-Rushood M (2015) Food Allergy: Clinical Management for General Pediatricians. Int J Pediatr Neonat Care 1: 107. doi: http://dx.doi.org/10.15344/ ijpnc/2015/107

Page 3 of 3

\section{Food allergy management}

Despite the increasing number and complexity of new diagnostic modalities, our general approach to food allergy remains unchanged. This involves allergen avoidance and emergency action plan.

\section{Allergen avoidance}

Strict allergen avoidance is the cornerstone of management. It is not easy for patients and families to adhere to this recommendation despite their willing and efforts. Studies have shown that accidental exposure occurs, especially in young children. Risk taking behavior is another important factor in adolescents. Strict avoidance involves label reading, identifying hidden products, communicating with restaurant staff and preventing cross contamination. Families should communicate with schoolteachers and nurses about their children's condition.

\section{Emergency action plan}

It should be discussed with patients and their caregivers that it is extremely important to carry Epinephrine auto injector (EAI). Teaching on how and when to use it should be offered at each clinic visit. It has been shown that repeated instructions are associated with better compliance. It is also invaluable to make sure that EAI is correctly used. It has been shown that EAI is not used during anaphylaxis by patients mostly because of fear [8].

The detailed management of anaphylaxis is not the aim of this review. However, it is essential to point out that intramuscular epinephrine is the first and the most important line of treatment. Delay or lack of administration is associated with fatal anaphylaxis, severe reactions and biphasic anaphylaxis. Another important risk factor for fatal anaphylaxis in pediatrics is the presence of asthma, especially if poorly controlled. Repeated epinephrine dosing should be used if the symptoms progress or the response is suboptimal.

Prompt recognition of anaphylaxis early manifestation as well as early administration of epinephrine is essential initial steps in the management of an acute reaction.

\section{Conclusion}

Food allergy is a growing health problem. It is vital to correctly be diagnosed. Overdiagnosis might compromise patient health and quality of life, while misdiagnosis can lead to fatal anaphylaxis. New modalities in the diagnosis and treatment are promising however, research to evaluate efficacy is needed. Primary prevention of food allergy is an important step in the management, the factors of which to be elucidated.

\section{Competing Interest}

The author declare that there is no competing interests exist.

\section{References}

1. NIAID-Sponsored Expert Panel, Boyce JA, Assa'ad A, Burks AW, Jones $\mathrm{SM}$, et al. (2010) Guidelines for the Diagnosis and Management of Food Allergy in the United States: Report of the NIAID-Sponsored Expert Panel. J Allergy Clin Immunol 126: S1-S58.

2. Bird JA, Lack G, Perry TT (2015) Clinical management of food allergy. J Allergy Clin Immunol Pract 3: 1-11.

3. Greenhawt M (2014) Food allergy quality of life. Ann Allergy Asthma Immunol 113: 506-512.

4. Sicherer SH, Leung DYM (2015) Advances in allergic skin disease, anaphylaxis, and hypersensitivity reactions to foods, drugs, and insects in 2014. J Allergy Clin Immunol 135: 357-367.

Int J Pediatr Neonat Care

ISSN: 2455-2364
5. Sichere SH, Sampson HA (2014) Food allergy: epidemiology, pathogenesis, diagnosis, and treatment. J Allergy Clin Immunol 133: 291-307.

6. Sampson HA (2003) Food allergy. J Allergy Clinlmmunol 111: s540-s547.

7. Simons FE, Ardusso LR, Bilò MB, El-Gamal YM, Ledford DK, et al. (2011) World health organization anaphylaxis guidelines: summary. J Allergy Clin Immunol 127: 587-593.

8. O'Keefe AW, De Schryver S, Mill J, Dery A, Ben-Shoshan M, et al. (2014) Diagnosis and management of food allergies: new and emerging options: a systematic review. J Asthma Allergy 7: 141-164.

9. Luengo O, Cardona V (2014) Component resolved diagnosis: when should it be used?. J clinTransl allergy 4: 1-9.

10. Sampson HA, Aceves S, Bock SA, James J, Jones S, et al. (2014) Food allergy: a practice parameter update-2014. J Allergy Clin Immunol. 134: 1016-1025.

11. Anagnostou K, Stiefel G, Brough H, du Toit G, Lack G, et al. (2015) Active management of food allergy: an emerging concept. Arch Dis Cild 100: 386390.

12. Peters RL, Gurrin LC, Allen KJ (2012) The predictive value of skin prick testing for challenge-proven food allergy: a systematic review. Ped Allergy Immunol 23: 347-352. 